history of hazardous alcohol consumption; and only a single individual within the 31-35 group had a fibroscan $>11 \mathrm{kPa}$ in absence of hazardous alcohol ingestion or co-infectivity with a hepatotropic virus.

Conclusion Going forward we therefore propose that those patients $<30$ years old do not necessitate elastography prior to treatment. The vast majority of patients between 30-35 years ( $>95 \%$ in our dataset) are also likely to be suitable for treatment without elastography in the absence of other coexistent factors for fibrosis; most notably alcohol in this cohort.

\section{PTU-006 VITAMIN D DEFICIENCY DOES NOT INCREASE THE RISK OF POST-LIVER TRANSPLANT ALLOGRAFT CELLULAR REJECTION (ACR)}

${ }^{2}$ See Chai Carol Chan, ${ }^{1}$ Freya Rhodes*, 'Olivia Greenham, 'Emma Hunt, ${ }^{1}$ Rachel H Westbrook. 'Royal Free NHS Foundation Trust, London, UK; ${ }^{2}$ University College London, London, UK

\subsection{6/gutjnl-2019-BSGAbstracts.215}

Introduction Vitamin D deficiency is prevalent in cirrhotic patients, and is increasingly associated with undesirable outcomes including increased rates of infection and higher mortality rates. It has also been documented that, owing to its supposed role in immune regulation, there may be a correlation between vitamin D deficiency pre-liver transplant and an increased risk of graft rejection (independent of other factors such as CMV rates which influence rejection risk). It has been postulated that this correlation may be because vitamin D allows a level of immune tolerance towards the graft. Nevertheless, the evidence is not conclusive. Animal models have demonstrated that calcitriol administration can prevent ACR and improve survival, but this has not yet been trialled in humans. Few retrospective studies have also looked at correlations between vitamin D deficiency and ACR rates- with differing results. We aimed to review outcomes of patients undergoing liver transplant at a tertiary liver centre, and evaluate if any such correlation with vitamin D and graft rejection exists.

Methods This is a retrospective study of patients with chronic liver disease awaiting liver transplantation from 2015-2017. Data collated included: demographic data, vitamin D levels within 6 months pre-liver transplant, use of vitamin D supplementation, post-operative rates of CMV, and episodes of cellular rejection (biopsy-proven).

Results 299 patients were included (72\% male). Aetiology was ALD $(n=96)$, viral hepatitis $(n=66), \quad$ PBC/PSC $(n=62)$, NAFLD $(n=34)$, AIH $(n=22)$, and 'other' $(n=19)$. Vitamin D deficiency affected $62 \%$ of the cohort. 157/299 patients had so far been transplanted at the time of data collection, and of these $115 / 157$ had vitamin D levels recorded within 6 months pre-transplant. Total rejection rates were 20\% (23/ $115)$ in the first year post-transplant, with the majority being episodes of early rejection (0-2 months). There was no significant association between vitamin $\mathrm{D}$ deficiency pre-transplant and the risk of cellular rejection, with $25 \%$ of those with vitamin $\mathrm{D}$ levels $<25 \mathrm{nmol} / \mathrm{L}$ pre-transplant experiencing rejection, in comparison to $14 \%$ of those with a normal vita$\min \mathrm{D}(>/ 50 \mathrm{nmol} / \mathrm{L})(\mathrm{p}=0.2)$. No correlation was seen with vitamin $\mathrm{D}$ and $\mathrm{CMV}$ rates, or $\mathrm{CMV}$ and rejection rates. Prescribing cholecalciferol either pre-transplant or in the first month post-transplant did not confer any significant reduction in the risk of rejection.

Conclusion Our results are contrary to the limited published data showing increased rates of graft rejection with vitamin D deficiency. There is a pressing need for a prospective study to confirm if a true correlation exists between vitamin D deficiency and risk of cellular rejection, and if vitamin D supplementation would reduce this risk.

\section{PTU-007 THE DEVELOPMENT OF OBESITY AND TYPE 2 DIABETES HARNESSING THE GUT MICROBIOME AND HOST GENETICS}

${ }^{1}$ Jocelyn Cheuk*, 'Sylvia Pender, ${ }^{2}$ Felino Cagampang. 'Faculty of Medicine, University Of Southampton, Southampton, UK; ${ }^{2}$ Human Development and Health, Institute of Developmental Sciences, Faculty of Medicine, Southampton, UK

\subsection{6/gutjnl-2019-BSGAbstracts.216}

Introduction Obesity is increasingly prevalent worldwide and has deleterious consequences on our health, including type 2 diabetes. Risk factors of obesity include genetic factors, diet, lifestyle and the gut microbiome. A preliminary study that combined genetic and environmental manipulations observed that MMP28 gene-knockout (MMP28KO) mice exhibit signs of obesity and type 2 diabetes. Interestingly, the metabolic profile of MMP28KO mice significantly improved upon transferring them from a specific pathogen free (SPF) area into the conventional mouse room for 5 weeks, suggesting the involvement of the gut microbiome. It is hypothesised that the underlying mechanisms are due to modulations in the insulin and Farnesoid X Receptor signalling pathways, which play important roles in regulating glucose and lipid metabolism. Key components within the pathways include Insulin receptor substrate-1 (IRS-1), insulin receptor substrate-2 (IRS-2) and Farnesoid X Receptor (FXR).

Aims This study aims to investigate the underlying mechanisms linking the MMP28 gene knockout effect and the impact of changing environments with changes in metabolic profiles.

Methodology A total of 71 mice were used in this study. Quantitative real time polymerase chain reaction was used to compare the expression levels of the IRS-1, IRS-2 and FXR genes between MMP28KO mice and their wild-type counterparts at 15 and 30 weeks of age. To investigate the impact of changing environments, the change in gene expression levels after the MMP28KO mice were moved from the SPF area into the conventional mouse room for 5 weeks was determined. Parametric data were compared using the unpaired ttest; non-parametric data were compared using the MannWhitney $U$ test. A $p$ value of $<0.05$ was considered significant.

Results The MMP28 gene knockout effect resulted in a poor metabolic profile through lowered IRS-2 and FXR gene expressions in the liver and pancreas $(p<0.05)$ in 30 -week-old mice. IRS-1 gene expression was also lower in the pancreas $(\mathrm{p}<0.05)$. Transferring MMP28KO mice from the SPF area to a conventional mouse room increased IRS-2 gene expression in both the liver and pancreas $(p<0.001$ at 30 weeks and $\mathrm{p}<0.05$ at 15 weeks, respectively). FXR gene expression only increased in the pancreas $(\mathrm{p}<0.05)$ in 15 -week-old mice and there were no changes in IRS-1 gene expression in either organ. The gene expression modulations of the two pathways in the liver and pancreas are summarised in figure 1. 